\title{
Assessment of diaphragm paralysis with oesophageal electromyography and unilateral magnetic phrenic nerve stimulation
}

\author{
Y.M. Luo, M.L. Harris, R.A. Lyall, A. Watson, M.I. Polkey, J. Moxham
}

\begin{abstract}
Assessment of diaphragm paralysis with oesophageal EMG and unilateral magnetic phrenic nerve stimulation. YM Luo, ML Harris, RA Lyall, A Watson, MI Polkey, J Moxham. (C) ERS Journals Ltd 2000.

ABSTRACT: The purpose of this study was to establish a sensitive and reliable method of diagnosing diaphragm paralysis by recording the diaphragm compound muscle action potential (CMAP) using a multipair oesophageal electrode and unilateral magnetic phrenic nerve stimulation.

An oesophageal electrode catheter was designed containing six coils $(1 \mathrm{~cm}$ wide and $3 \mathrm{~cm}$ apart), creating an array of four sequential electrode pairs. The oesophageal catheter was taped at the nose with the proximal electrode pair $40 \mathrm{~cm}$ from the nares. Eight patients with unilateral $(n=5)$ or bilateral $(n=3)$ diaphragm paralysis were studied. Five to seven phrenic nerve stimulations were performed at $80 \%$ of maximum magnetic stimulator output and the CMAPs were recorded simultaneously from the four pairs of electrodes.

In the five patients with unilateral diaphragm paralysis, the CMAP amplitudes and latencies were $1.16 \pm 0.29 \mathrm{mV}$ and $7.6 \pm 1.5 \mathrm{~ms}$ for functioning sides. No diaphragm CMAP could be detected when stimulating nonfunctioning phrenic nerves.

This study shows that diaphragm paralysis can be reliably diagnosed by unilateral magnetic stimulation combined with a multipaired oesophageal electrode. Eur Respir J 2000; 15: 596-599.
\end{abstract}

\author{
Dept of Respiratory Medicine and Allergy, \\ Guy's, King's and St Thomas' School of \\ Medicine, King's College Hospital, Lon- \\ don, UK. \\ Correspondence: Y.M. Luo \\ Respiratory Muscle Laboratory \\ King's College School of Medicine \& \\ Dentistry \\ Bessemer Road \\ London SE5 9PJ \\ UK \\ Fax: 442073463589
}

Keywords: Diaphragm electromyography diaphragm paralysis

phrenic nerve stimulation

Received: June 131999

Accepted after revision November 121999
There is no ideal method of reliably diagnosing diaphragm paralysis [1], although a number of techniques have been employed, such as fluoroscopy of the diaphragm, measurement of transdiaphragmatic pressure and ultrasound evaluation. Recording the diaphragm compound muscle action potential (CMAP) during electrical stimulation of the phrenic nerve (ES) remains an important technique [2], although, in some patients, for example those who are obese, ES can be difficult. Unilateral magnetic phrenic nerve stimulation (UMS) is an easy and reliable method of supramaximally stimulating the phrenic nerve, but the diaphragm CMAP recorded from chest wall surface electrodes can be contaminated by the coactivation of other chest wall muscles [3-6]. The diaphragm CMAP recorded from an oesophageal electrode is less contaminated [3, 7]. However, conventional single-pair oesophageal electrodes can be difficult to optimally position at the diaphragm electrical activity region (EARdi) [8] and an absent CMAP in response to phrenic nerve stimulation can be simply due to the recording electrode being distant from the diaphragm. To reliably record the diaphragm CMAP, a multipair oesophageal electrode was used to overcome the difficulty of positioning the electrode at the EARdi. The aim of the study was to develop a method of reliably diagnosing diaphragm paralysis by combined use of UMS and multipair oesophageal electrodes.

\section{Methods}

Eight patients with unilateral (five patients) or bilateral (three patients) diaphragm paralysis, based on the finding of elevation of the diaphragm on chest radiography or paradoxical movement on fluoroscopy plus an absent CMAP response to ES recorded with chest wall electrodes, were studied. Unilateral and bilateral diaphragm paralysis were further confirmed by sniff transdiaphragmatic pressure (Pdi) and the Pdi response to bilateral magnetic stimulation and UMS. The cause of diaphragm paralysis was undetermined in four patients (Nos. 1,2,3 and 4). Two of the remaining four had neuralgic amyotrophy (Nos. 6 and 8), one amyotrophic lateral sclerosis (No. 7) and one phrenic nerve trauma (No. 5). The patients with bilateral diaphragm paralysis and three of the patients with unilateral diaphragm paralysis (Nos. 2, 3 and 4) had severe or moderate breathlessness. The study was approved by the ethics committee of King's College Hospital and patients gave informed consent.

UMS was performed using a 43-mm double coil $(\mathrm{P} / \mathrm{N}$ 9784-00; Magstim Co. Ltd., Whitland, Dyfed, UK) powered by a Magstim 200 stimulator (Magstim Co., Whitland, Dyfed, UK). The coil was placed anterolaterally on the neck over the phrenic nerve [3]. Subjects were seated upright in a chair and stimulation was performed at resting 
end-expiration. Five twitches were obtained at $80 \%$ of magnetic stimulator output.

Oesophageal electrodes consisted of six copper coils, which were $1 \mathrm{~cm}$ in length and separated by a distance of 3 $\mathrm{cm}$. The top coil was connected to earth and the lower five coils formed four sequential pairs of electrodes with a span of $17 \mathrm{~cm}$ (fig. 1). The oesophageal electrode was passed through the nose and swallowed into the oesophagus. Recordings were performed with the proximal electrode pair $40 \mathrm{~cm}$ from the nostril in all subjects. Electromyographic (EMG) signals recorded from the four electrode pairs were amplified and band-pass filtered between $10 \mathrm{~Hz}$ and 10 $\mathrm{kHz}$ (Magstim Co.). The signals were then passed to a 12bit analogue-to-digital converter (NB-MIO-16; National Instruments, Austin, TX, USA) and stored and displayed on a Macintosh Centris Computer running Labview 2.2 software (National Instruments).

The diaphragm CMAP amplitude was measured from peak to peak. The latency of the CMAP was defined as the time from the stimulation artefact to the onset of the
CMAP. To eliminate the influence of the electrocardiogram (on the oesophageal electrode) and oesophageal peristalsis (i.e. avoiding QRS waves of the electrocardiogram during analysis of the EMG), only CMAPs with a constant shape and a stable baseline before and after stimulation were analysed. Unless stated otherwise, the data reported were measured from the electrode pair with greatest CMAP amplitude. Results are expressed as the mean $\pm \mathrm{SD}$.

\section{Results}

The oesophageal electrode was well tolerated by all patients. No diaphragm CMAP could be recorded with UMS on either side in the two (Nos. 6 and 7) of three patients diagnosed as having bilateral diaphragm paralysis. In one patient (No. 8) who was diagnosed as having bilateral diaphragm paralysis, no CMAP was recorded from the left side; however, a small CMAP with a prolonged latency was observed on the right side. No diaphragm CMAP could be recorded on one side in the five patients with unilateral diaphragm paralysis, whereas a clear CMAP was a)

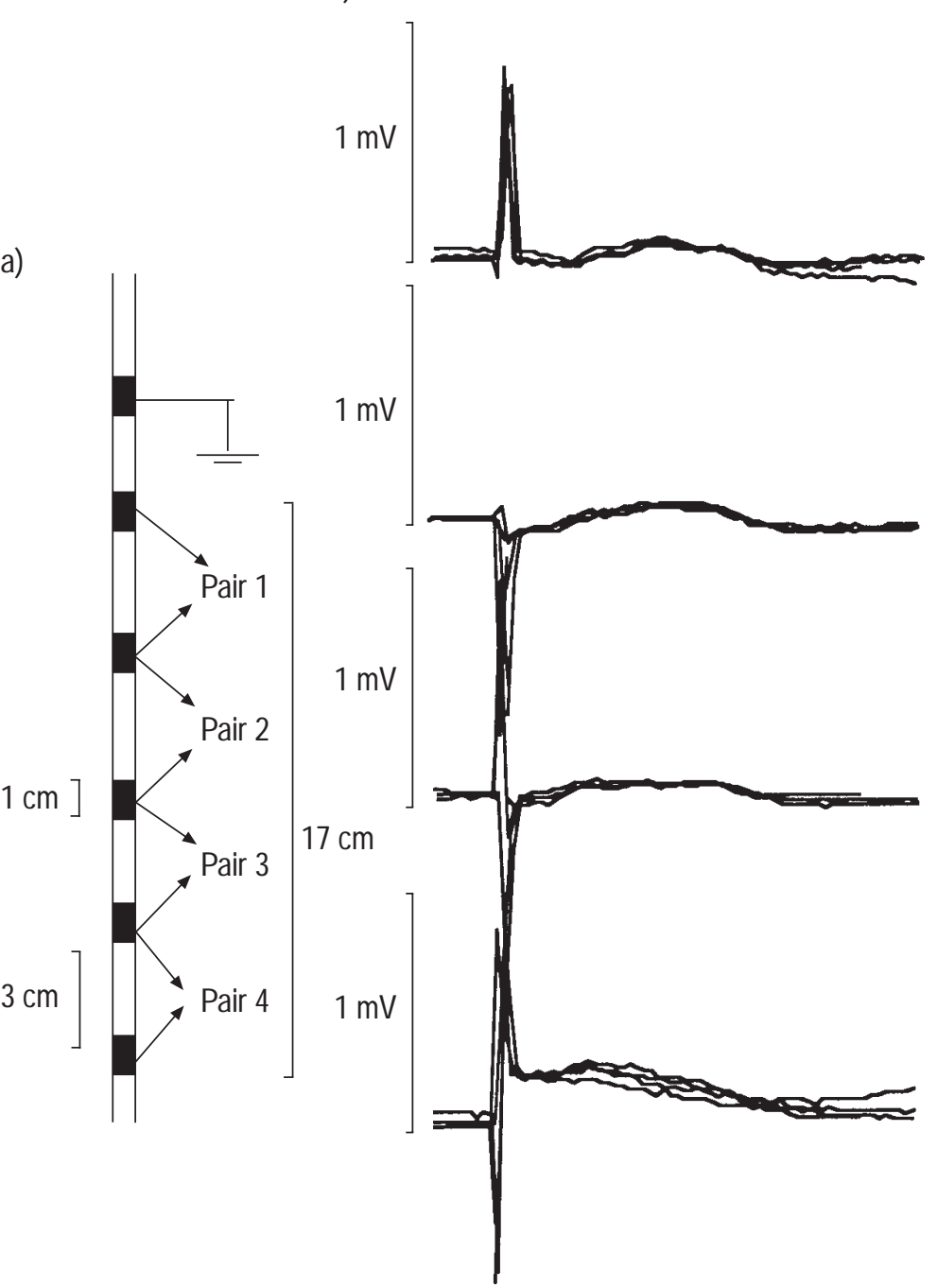

c)
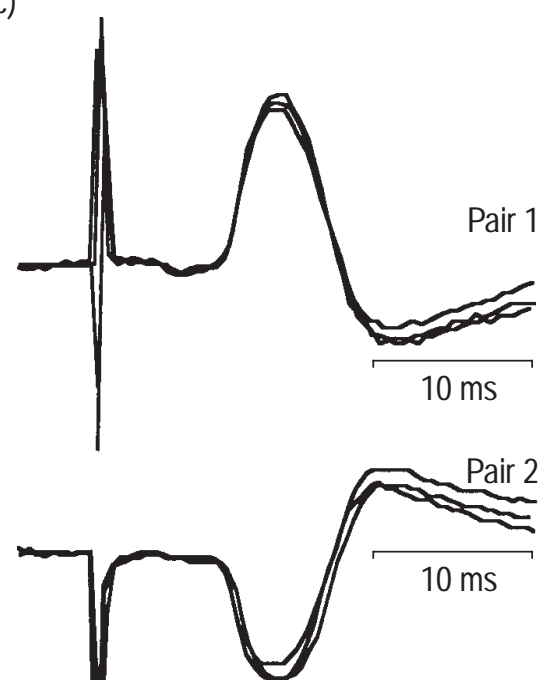

Pair 3

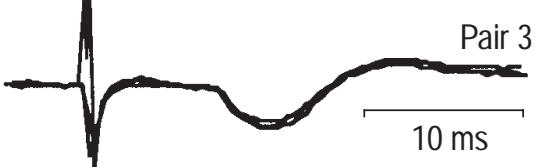

Pair 4

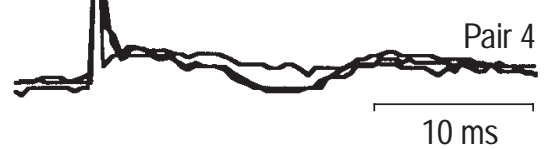

Fig. 1. - Multipair oesophageal electrode. Each electrode is $1 \mathrm{~cm}$ wide and there is a $3 \mathrm{~cm}$ distance between electrodes. The top electrode is connected to earth, and the lower five create four consecutive pairs of electrodes. b, c) Diaphragm compound muscle action potential (CMAP) elicited by unilateral magnetic phrenic nerve stimulation in unilateral diaphragm paralysis: b) no CMAP was recorded from the left side side (nonfunctioning phrenic nerve); and c) CMAP recorded from the right side. The data represent three superimposed signals from patient 4. 
recorded on the normal side. The CMAP amplitude and latency in these patients were $1.16 \pm 0.29 \mathrm{mV}$ and $7.6 \pm 1.5$ $\mathrm{ms}$ (pooled left and right values) for functioning hemidiaphragms (table 1, fig. 1).

\section{Discussion}

$\mathrm{ES}$ is an important technique in assessing diaphragm paralysis. However, it can be difficult to perform. The time taken to locate the phrenic nerve with ES can be up to 20 min [9]. Mier et al. [9] found that the phrenic nerve could not be located with ES in some subjects, especially patients with diaphragm weakness. Repeated stimulation for $30 \mathrm{~min}$ has been required to establish a diagnosis of diaphragm paralysis with reasonable confidence in the absence of a CMAP response to ES [9]. Once the phrenic nerve is located, it can be difficult; with ES, to sustain maximal stimulation because the position and direction of the stimulating electrode is critical, whatever the stimulus intensity. In contrast, UMS uses a large surface area for stimulation and the phrenic nerve can be easily and reliably stimulated [3]. Because it is easy to apply and maximally stimulates the phrenic nerve, UMS is likely to be useful in clinically assessing patients with diaphragm paralysis. However, UMS coactivates the brachial plexus, which can make it difficult to measure the diaphragm CMAP with chest wall electrodes because of signal contamination $[3,5,6]$. It has been shown that the diaphragm CMAP recorded from chest wall electrodes is unreliable when the brachial plexus is activated [6]. Therefore, to take advantage of UMS, it is necessary to record the diaphragm CMAP with an oesophageal electrode since it is less contaminated by extradiaphragmatic muscle activity $[3,6]$.

It can be difficult to accurately position a single-pair electrode at the EARdi and repositioning of the electrode may be necessary, which is time-consuming and uncomfortable. Positioning the electrode by recording the voluntary EMG during quiet breathing is not always reliable because the signal-to-noise ratio is low, especially in patients with phrenic nerve dysfunction. Positioning the electrode based on maximal voluntary EMG may not be satisfactory for recording the diaphragm CMAP at resting end-expiration since the EARdi centre may move [10]. A balloon at the end of a single pair electrode wedged near the gastro-oesophageal junction can help in positioning the electrode [10]. However, the technique can be uncomfortable and the balloon can be inadvertently drawn back into the oesophagus. With the multipair electrode, the EARdi centre can always be adequately covered with the catheter taped at the nose $40 \mathrm{~cm}$ from the first electrode pair and the recording electrodes $40 \mathrm{~cm}$ proximal to the nose, to $57 \mathrm{~cm}$ distal from the nose. McKenzIE and GANDEVIA [7] showed that the electrode position in the oesophagus for optimal recording of the diaphragm CMAP was $45-55 \mathrm{~cm}$ from the nose, a span easily covered by the multipair electrode. The reversed polarity of the diaphragm CMAP in consecutive pairs (fig. 1) further confirms that the electrode pairs cover the EARdi centre $[3,5,8]$. It would be expected that the amplitude of the CMAP measured with a $3-\mathrm{cm}$ interelectrode distance (in the present study) would be smaller than that measured using one of 6-cm, as used by McKenZIE and GANDEVIA [7], since the longer the distance between the electrodes the greater the amplitude of the CMAP [7]. However, the maximal CMAP amplitude measured using the multipair electrode in the present study was comparable to that measured with a $6-\mathrm{cm}$ interelectrode distance, suggesting that one of the pairs is at or very closed to the EARdi centre. Thus, the diaphragm CMAP can be reliably recorded by the multipair oesophageal electrode and, because positioning has been simplified and UMS is easy, reliable diagnosis of diaphragm paralysis can be achieved. This method may be useful in most clinical situations, including investigations in obese patients, and those with a neck catheter in the intensive care unit.

In conclusion, this study demonstrates that the method involving unilateral magnetic phrenic nerve stimulation and a multipair oesophageal electrode is a reliable and sensitive technique for diagnosing diaphragm paralysis. When the diagnosis of diaphragm paralysis cannot be made with confidence using surface recordings of the diaphragm compound muscle action potential and electrical stimulation of the phrenic nerve, the method utilizing unilateral magnetic phrenic nerve stimulation and a multipair oesophageal electrode could be helpful.

\section{References}

1. Gottesman E, McCool FD. Ultrasound evaluation of the paralysed diaphragm. Am J Respir Crit Care Med 1997; 155: $1570-1574$.

2. Moorthy SS, Markand ON, Mahomed Y, Brown JW. Electrophysiologic evaluation of phrenic nerves in severe respiratory insufficiency requiring mechanical ventilation. Chest 1985; 88: 211-214.

Table 1. - Diaphragm compound muscle action potential

\begin{tabular}{|c|c|c|c|c|c|c|c|c|}
\hline \multirow{2}{*}{ Patient No. } & \multirow{2}{*}{ Sex } & \multirow{2}{*}{ Age yrs } & \multirow{2}{*}{ Height $\mathrm{cm}$} & \multicolumn{2}{|c|}{ Amplitude mV } & \multicolumn{2}{|c|}{ PNCT ms } & \multirow{2}{*}{ Side involved } \\
\hline & & & & Left & Right & Left & Right & \\
\hline 1 & M & 40 & 175 & 0 & 0.98 & - & 6.9 & $\mathrm{~L}$ \\
\hline 3 & $\mathrm{~F}$ & 63 & 161 & 0 & 1.45 & - & 6.3 & $\mathrm{~L}$ \\
\hline 3 & M & 60 & 165 & 0 & 0.91 & - & 8.4 & $\mathrm{~L}$ \\
\hline 4 & M & 47 & 174 & 0 & 0.97 & - & 9.8 & $\mathrm{~L}$ \\
\hline 5 & M & 14 & 172 & 1.50 & 0 & 6.6 & - & $\mathrm{R}$ \\
\hline 6 & M & 74 & 174 & 0 & 0 & - & - & $\mathrm{Bi}$ \\
\hline 7 & M & 77 & 155 & 0 & 0 & - & - & $\mathrm{Bi}$ \\
\hline 8 & M & 35 & 201 & 0 & 0.14 & - & 14.0 & $\mathrm{Bi}$ \\
\hline
\end{tabular}

PNCT: phrenic nerve conduction time; M: male; F: female; L: left; R: right; Bi: bilateral. 
3. Luo YM, Johnson LC, Polkey MI, et al. Diaphragm electromyogram measured with unilateral magnetic stimulation. Eur Respir J 1999; 13: 385-390.

4. Mills GH, Kyroussis D, Hamnegard CH, Wragg S, Moxham J, Green M. Unilateral magnetic stimulation of the phrenic nerve. Thorax 1995; 50: 1162-1172.

5. Luo YM, Polkey MI, Johnson LC, et al. Diaphragm EMG measured by cervical magnetic and electrical phrenic nerve stimulation. J Appl Physiol 1998; 85: 2089-2099.

6. Luo YM, Polkey MI, Lyall RA, Moxham J. Effect of brachial plexus coactivation on phrenic nerve conduction time. Thorax 1999; 54: 765-770.

7. McKenzie DK, Gandevia SC. Phrenic nerve conduction times and twitch pressures of the human diaphragm. $J$ Appl Physiol 1985; 58: 1496-1504.

8. Beck J, Sinderby C, Lindstrom L, Grassino A. Diaphragm interference pattern EMG and compound muscle action potentials: effects of chest wall configuration. $J$ Appl Physiol 1997; 82: 520-530.

9. Mier A, Brophy C, Moxham J, Green M. Phrenic nerve stimulation in normal subjects and in patients with diaphragmatic weakness. Thorax 1987; 42: 885-888.

10. Gandevia SC, McKenzie DK. Human diaphragmatic EMG: changes with lung volume and posture during supramaximal phrenic stimulation. J Appl Physiol 1986; 60: $1420-1428$. 